Acta Crystallographica Section B

Structural

Science

ISSN 0108-7681

\section{Angel Vegas ${ }^{\mathrm{a} *}$ and Valentín García-Baonza $^{\text {b }}$}

'Instituto de Química Física 'Rocasolano', CSIC, C/Serrano 119, 28006 Madrid, Spain, and

b Departamento de Química Física, Facultad de Quimicas, Universidad Complutense, 28040

Madrid, Spain

Correspondence e-mail: avegas@iqfr.csic.es

\title{
Pseudoatoms and preferred skeletons in crystals
}

The generalization of the Zintl-Klemm concept provides a universal formulation of a crystal structure in terms of universal building skeletons formed by Klemm's pseudoatoms: atoms that behave structurally according to their formal total electron charge. An important difference in this novel view is that charge is considered to be transferred, in the strict Zintl's sense, from the donor cations to the building skeleton as a whole, not specifically to a given atom or ion. Although application is restricted to (IV)-(IV) compounds (group 14 structures), the principle seems to be universal and can be applied to understand, to relate and to predict the structure of complex compounds on the basis of more simple structures, e.g. a given $A B$ skeleton provides the building block for $A_{2} B, A B_{2}, A B X_{m}$ etc. compounds of a very different nature. The application of such a principle only requires information on the constituent atoms and on the existing phases of the $p$-block elements (observed under ambient and high-pressure and/or high-temperature conditions). The ideas introduced here demonstrate, for the first time, that a generalization of the Zintl-Klemm concept is possible and that such a generalization helps to establish a univocal link between chemical composition (in terms of pseudoatoms) and the crystalline structures observed experimentally.

\section{Introduction}

The great challenge, as yet unmet, of solid-state chemistry is the understanding of the fundamental principles governing the formation of crystal structures. It is generally admitted that the rules enumerated in the 1930s, which are based almost exclusively on atomic sizes and electrostatic forces, have not allowed either the understanding or the prediction of crystalline structures. Moreover, their description has been, in many instances, a rather cumbersome task.

Despite the astonishing developments of computational methods and their impact in the prediction of a number of properties of crystals (mechanical, electrical etc.), first-principles calculations were not able to predict crystal structures, since an initial set of candidate structures is always required before performing a geometry optimization. In binary compounds, possible alternative structures can be proposed, for instance, from the empirical Pettifor structure maps (Simak et al., 1997). The works of Jansen and co-workers (Schön \& Jansen, 1996; Jansen, 2002) have shown the first steps towards prediction and synthesis planning. More recent studies have demonstrated that a combination of data mining and quantum mechanical calculations (Fischer et al., 2006), and the use of evolutionary algorithms (Hart et al., 2005; Oganov \& Glass, 2006) provide appealing alternatives for structure prediction. In the case of data-mining-based
Received 26 February 2007 Accepted 17 April 2007
(C) 2007 International Union of Crystallography Printed in Singapore - all rights reserved 
methods, the increasing information included in structural databases helps to ease the task. In any case, it should be emphasized that even if some kind of prediction is currently feasible (Schön \& Jansen, 1996; Jansen, 2002), the crucial aspect, i.e. the understanding of the crystalline state, is still at a primitive level. In our opinion, one of the reasons for this poor advancement in the understanding of crystal formation concerns the almost unique accepted way of looking at crystal structures: the so-called cation-centred anions polyhedra. Only in the last few decades have attempts to develop alternative approaches to describe the structures of crystals been made. Among others, we mention the ideas introduced by O'Keeffe \& Hyde (1985), Borisov (2000), and Vegas \& coworkers (Vegas, 2000; Vegas \& Jansen, 2002; SantamaríaPérez \& Vegas, 2003; Santamaría-Pérez et al., 2005; Vegas et al., 2006), who have provided new insights into the analysis of the cation arrays in oxides. These works have led to an important conclusion: cations seem to govern the skeletons of crystals. The most recent works are especially illuminating in this regard, since these authors succeeded in rationalizing and explaining the structures of aluminates and silicates by applying the Zint-Klemm concept (ZKC; Zintl \& Dullenkopf, $1932)$ to the cation arrays of these families of oxides (Santamaría-Pérez \& Vegas, 2003; Santamaría-Pérez et al., 2005). These somewhat surprising results indicate that cations in silicates/aluminates behave as real Zintl phases and that the aluminates/silicates polyanions are real Zintl polyanions in which the $\mathrm{O}$ atoms are located in the vicinity of both bonding and lone pairs.

The Zintl-Klemm concept, one of the most simple but powerful concepts in solid-state chemistry, is also among the most widely accepted in different areas of chemistry. Such a concept was introduced to rationalize the structures of the later so-called Zintl phases. Typical Zintl compounds are formed when a very electropositive element reacts with a $p$ block element with medium electronegativity. The ZKC requires the formal charge transfer of the valence electrons from the electropositive element to the electronegative. The negatively charged atoms (the so-called Zintl polyanions) form partial structures with directed bonds obeying the ' 8 -N rule' that are similar to the structures found in the (neutral) main-group elements having the same number of valence electrons. Although the ZKC is mainly applied to intermetallics (Zintl phases), the issue of extending the ZintlKlemm concept to transition metals (assuming that transition metals act electropositively; Kauzlarich, 1996), anionic transition metals (Karpov et al., 2004) and the aforementioned example of aluminates and silicates has been addressed.

The present work represents a step forward in the generalization of the $\mathrm{ZKC}$, as our goal here is to demonstrate that the ZKC provides the key to understand more complex structures, provided that a formal charge transfer between cations takes place in the crystal. In other words, it appears that the ZKC concept is more universal than previously thought.

Our initial motivation in the present study was to understand the structural correlations observed for different families of homologous compounds (wide sense), in an attempt to generalize the so-called anions on a metallic matrices (AMM) model recently developed (Vegas et al., 2006; Marqués et al., 2006). This model, which gave a successful interpretation of the structures of silicates and aluminium halides, provides a direct interpretation of the particular structures shown by oxides by considering that the crystal structure can be understood as a metallic matrix (or Zintl polyanion) acting as a host lattice for the non-metallic atoms, the formation and localization of the anions in the compound being driven by the geometric and electronic structures of the metallic sub-structure.

\section{Preferred skeletons}

After the analysis of several hundred compounds, we found that a number of preferred skeletons (PS) appeared in a recurrent fashion either in the structure itself (in binary and ternary alloys) or in the cation subarrays (in ternary and quaternary oxides). We also found that the number of such PS was certainly limited to those of the $p$-block elements, specifically those of Group 14. We soon realised that the cationic substructures of oxides could be rationalized in terms of the PS exhibited by binary alloys. Furthermore, all the cationic substructures in the oxides could also be explained in terms of the ZKC. Thus, if we assume that a generalization of the ZKC holds, a universal principle seems to work as follows: In any oxide, a pair of cations tends to produce skeletons characteristic of the $p$-block elements in such a way that the valence electron count (VEC) is satisfied and the 8-N rule is maintained.

As already noted above, it appears that the PS are those of Group 14, as a more or less homogeneous charge distribution would be a stability factor for a given structure. The analysis of the relative occurrence of a given PS reveals that those derived from (IV)-(IV)-type compounds (diamond, blende, $\mathrm{SrAl}_{2}$ etc.) are certainly quite common. Thus, it seems that if the number of valence electrons that can be shared by the structure completes an octet, the compound tends to acquire a PS of an element of Group 14. It therefore appears that some sort of '(IV)-(IV) rule' holds in the formation of a given compound [a well known fact in (IV)-(IV), (III)-(V) and (II)(VI) binary semiconductors]. Thus, whenever possible, a pair of cations tends to acquire a full-octet configuration, giving rise to a (IV)-(IV)-like structure. If the number of valence electrons is smaller than eight, it appears that a pair of cations tend to acquire electron charge from others present in the structure whatever type it is (i.e. they can be of the same kind) to fulfill the octet configuration. In order to achieve this, the necessary formal electron transfer between cations seems to be produced to form what we have named the building skeleton (BS).

Within this view, the BS is considered to be formed by pseudoatoms in the original Klemm's sense, i.e. atoms that behave structurally according to their formal total electron charge (pseudoatoms will be labelled with the $\Psi$ prefix in the following). As a result, we propose that a given structure 
should be universally formulated as $D_{n}[\mathrm{BS}] X_{m}$, where $D$ is the donor cation, the BS cations can be regarded as cations $e x$ officio (Parthé \& Engel, 1986; Parthé \& Chabot, 1990) and, if present, $X$ represents the anion. An important outcome of this view is that charge can be transferred, in Zintl's sense, to the $\mathrm{BS}$ as a whole and not specifically to a given atom or ion. To illustrate this idea we shall consider the example of $\mathrm{Na}_{3} \mathrm{P}$, where two $\mathrm{Na}$ atoms formally donate charge to the $(\mathrm{NaP})^{2-}$ entity (namely $\Psi$-AlP) that forms graphite-like layers (typical of the group 14 elements) with two $\mathrm{Na}^{+}$cations (closed-shell-like) intercalated between them. Accordingly, $\mathrm{Na}_{3} \mathrm{P}$ should be reformulated as $\mathrm{Na}_{2}[\mathrm{NaP}]$.

In order to demonstrate the general applicability of the generalized ZKC we shall first analyse three binary structures: $\mathrm{ZnS}$ (blende), NiAs and MnP, and related structures. The prototypical structure of blende [a (II)-(VI) compound] can

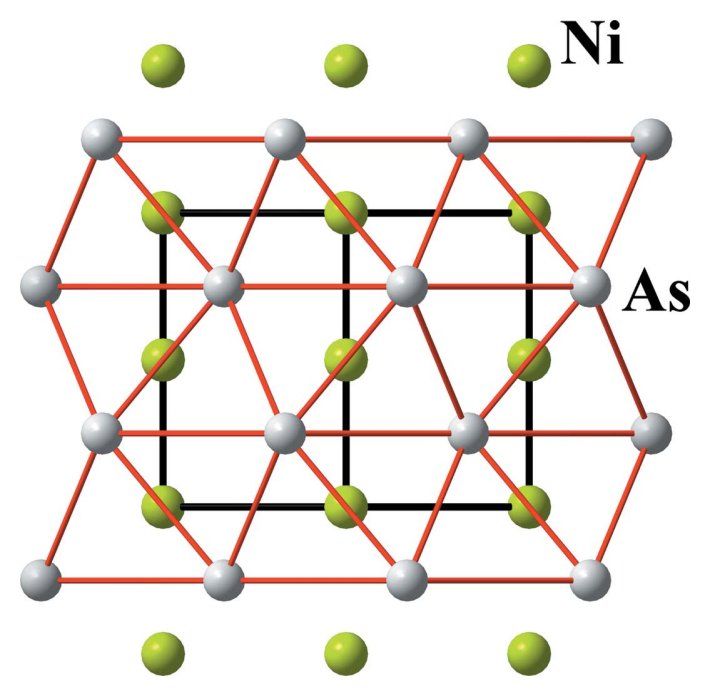

Figure 1

The NiAs structure viewed along [110]. The As atoms (grey circles) are connected by lines to depict the octahedra filled with the $\mathrm{Ni}$ atoms (green).

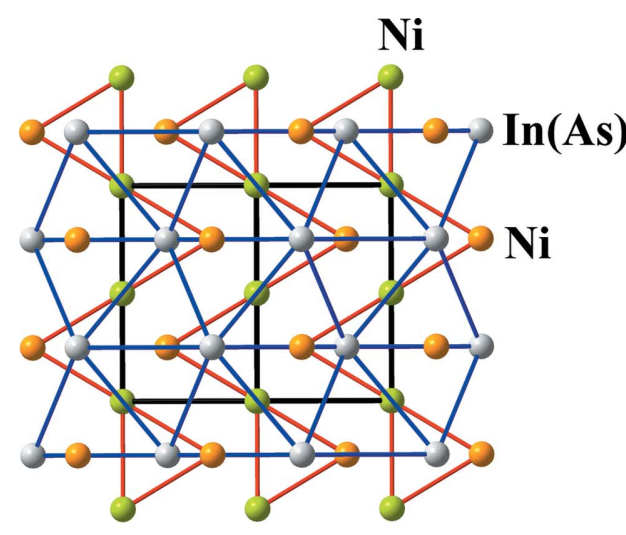

(a)

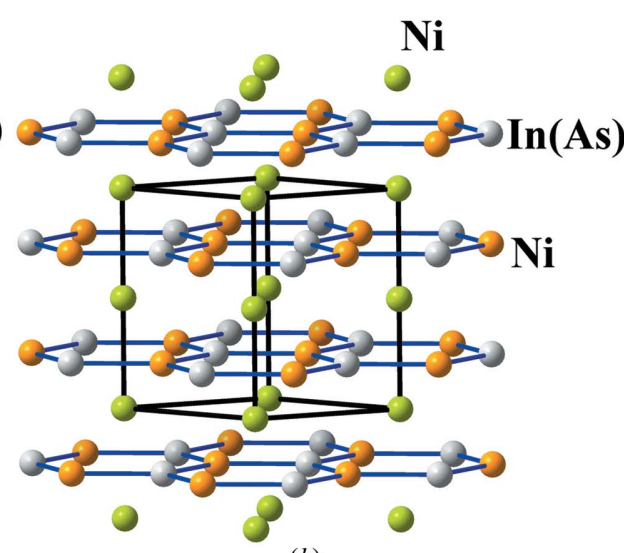

(b)
Figure 2

(a) The NiAs structure of Fig. 1 viewed along [110] stuffed with an additional Ni atom (orange) to form the $\mathrm{Ni}_{2}$ In structure. The blue contacts form the As octahedra shown in red in Fig. 1. The red contacts represent the trigonal prisms of $\mathrm{Ni}$ atoms which are occupied by the As atoms. (b) Perspective view of the same structure in which the graphite-like NiAs layers are highlighted. be considered as a substructure of diamond. It is well known that many other ternary compounds, like the prototypical Zintl compound LiAlSi, or MgAgAs, can be regarded as stuffed blendes. Early first-principles calculations in LiAlSi were unable to confirm the charge transfer from $\mathrm{Li}$ to $\mathrm{Al}$ (to convert it into a $\Psi$-Si) leading to a widespread critique of the Zintl model. The reader is referred to the article of SeifertLorenz \& Hafner (2002) for a discussion on the current understanding of the ZKC in terms of first-principles calculations. Both compounds LiAlSi and $\mathrm{MgAgAs}$ exhibit diamond-like skeletons BS, which are formed by (AISi) and (AgAs) entities, respectively. Assuming that the ZKC holds, these structures can be rationalized if one considers that the $\mathrm{Li}$ $(\mathrm{Mg})$ atoms formally provide one (two) electron(s) to the BS. While in the first case, the $\mathrm{BS}$ is well defined as $[\Psi-\mathrm{Si} \mathrm{Si}]$ or $[\mathrm{Al}$ $\Psi-\mathrm{P}]$ in terms of pseudoatoms, in the second case, given the capability of $\mathrm{Ag}$ to behave as a Zintl acceptor, it could be hypothesized that the electron donation from $\mathrm{Mg}$ turns the $\mathrm{Ag}$ atoms into $(\Psi-\operatorname{In})$. Thus, the BS ( $\Psi$-In) As forms a diamondlike structure with the BS atoms following the structural trend observed in (III)-(V) compounds. In fact, let us note again that InAs itself forms a blende-type structure. In any case, since at present we cannot determine whether charge is localized on a given atom, we prefer to refer to the BS as a whole.

The second structure to be considered is NiAs and its stuffed variant $\mathrm{Ni}_{2} \mathrm{In}$. NiAs is usually described as an h.c.p. (hexagonal close packing) of As atoms with all the octahedral voids occupied by Ni atoms (Fig. 1). An inverse description is a simple hexagonal array of $\mathrm{Ni}$ atoms with one half of the trigonal prisms occupied by As atoms (Fig. 2a). If the empty trigonal prisms are filled with additional atoms, the $\mathrm{Ni}_{2} \mathrm{In}$-type structure is produced. Such filling leads to a structure in which h.c.p. layers of $\mathrm{Ni}^{2+}$ cations alternate with graphite-like layers formed by (NiIn) ${ }^{2-}$ entities (Fig. 2b). Such an interpretation in terms of graphite-like layers was already considered by Zheng \& Hoffmann (1989). At this point, we provide a new reinterpretation of the $\mathrm{Ni}_{2} \mathrm{In}$ structure within the generalized ZKC, since the important question is why $\mathrm{Ni}_{2} \mathrm{In}$ forms such a structure. Within the generalized ZKC, the $\mathrm{Ni}$ atoms formally transfer two electrons to the BS, turning the In atoms into $\Psi$-Sb atoms, the final formula being $\mathrm{Ni}[\mathrm{NiIn}]$, with the BS [Ni $-\mathrm{Sb}]$ having the NiAstype structure, just the structure of $\mathrm{NiSb}$ itself. Thus, $\mathrm{Ni}_{2} \mathrm{In}$ is really an $\mathrm{Ni}$-stuffed NiAs structure and, to our knowledge, this is the first time that a simple and rational explanation is given for this compound.

A similar reasoning could be made for the MnP structure, which is intimately related to NiAs. $\mathrm{MnP}$ can be described as a distortion of 
NiAs in which the $\mathrm{P}$ atoms form a distorted h.c.p. array with all the octahedral voids occupied by Mn (Fig. $3 a$ ). In the same manner that $\mathrm{Ni}_{2} \mathrm{In}$ can be derived from $\mathrm{NiAs}$, the $\mathrm{Co}_{2} \mathrm{Si}$-type structure, and hence the cotunnite structure, can be derived from MnP. The reality is that MnP is the parent skeleton of a (vacant) cotunnite-like structure. If an additional atom is inserted into one half of the tetrahedral voids of $\mathrm{MnP}$, then the cotunnite structure is formed (Fig. 3a). The additional atom together with the $\mathrm{Mn}$ atoms form trigonal prisms where the $\mathrm{P}$ atoms are lodged (see Fig. 3b).

Summarizing, the following phase relations hold:

\begin{tabular}{lllll}
\hline Compound & Structure type & $\mathrm{D}+\mathrm{BS}$ & $\mathrm{BS}$ & BS structure \\
\hline $\mathrm{Ni}_{2} \mathrm{In}$ & $\mathrm{Ni}_{2} \mathrm{In}$ & $\mathrm{Ni}+\mathrm{NiAs}$ & $\mathrm{Ni} \Psi-\mathrm{Sb}$ & $\mathrm{NiAs}$ \\
$\mathrm{Mn}_{2} \mathrm{Ge}$ & $\mathrm{Ni}_{2} \mathrm{In}$ & $\mathrm{Mn}+\mathrm{MnGe}$ & $\mathrm{Mn} \Psi-\mathrm{Se}$ & $\mathrm{NiAs}$ \\
$\mathrm{Co}_{2} \mathrm{Si}$ & $\mathrm{Co}_{2} \mathrm{Si}$ (cotunnite) & $\mathrm{Co}+\mathrm{CoSi}$ & $\mathrm{Co} \Psi-\mathrm{P}$ & $\mathrm{MnP}$ \\
\hline
\end{tabular}

In fact, $\mathrm{Co}_{2} \mathrm{Si}$ (Fig. $3 b$ ) has the cotunnite structure. Interestingly, the ternary compounds MnNiSi and CoVSi have the same structure as $\mathrm{Co}_{2} \mathrm{Si}$, confirming the underlying transformations expressed in the above diagram. Let us note here that all the above structures present BS of the (IV)-(IV)-type, including the cotunnite structure. Thus, a diamond-like net is found in stuffed blendes, the $\Psi$-Si skeleton of $\mathrm{SrAl}_{2}$ is found in cotunnites, and graphite-like layers are characteristic of the $\mathrm{Ni}_{2}$ In-type structures. It is also important to point out that the transitions between these phases always involve the stabilization of Group 14 structures. The interesting conclusion of the above structural sequences is that they demonstrate how these structures convert into each other. As a matter of fact, the fluorite (stuffed blende) $\rightarrow$ cotunnite $\rightarrow \mathrm{Ni}_{2}$ In sequence has already been observed in several compounds (Vegas et al.,
2001). Furthermore, the NiAs $\rightarrow$ MnP phase transition also exists in FeS, a compound that provides an excellent example of the generalized ZKC involving phase transitions within the same compound. Thus, besides the NiAs and MnP structures, $\mathrm{FeS}$ also adopts the $\mathrm{PbO}$-type structure, a mineral known as mackinawite $(P 4 / \mathrm{nmm})$, which is also found in $\mathrm{BaO}$, BiIn, $\mathrm{CuFeTe}_{2}, \mathrm{FeSe}$, and in more than a hundred 2:1 compounds (e.g. $\mathrm{Mn}_{2} \mathrm{As}, \mathrm{Cr}_{2} \mathrm{As}, \mathrm{Fe}_{2} \mathrm{As}, \mathrm{NaMnAs}, \mathrm{CaMnGe}, \mathrm{Cu}_{2} \mathrm{Sb}$, among others). In all these compounds the PbO-like $\mathrm{BS}$ also fulfils the octet configuration, forming a four-connected net typical of (IV)-(IV) compounds. This is the case for the Zintl phase $\mathrm{NaAlSi}$, where the (AISi) ${ }^{-}$BS is identical to those found in the compounds listed above (in contrast to the stuffed blende structure adopted by the analogous LiAlSi). Finally, it is worth mentioning that in the ternary sulfide $\mathrm{KLiS}$, the $\mathrm{BS}(\mathrm{LiS})^{-}$is of the same type. It should be pointed out that the structure of $\mathrm{KLiO}$ was interpreted assuming that the $\mathrm{K}$ atom donates its valence electron to the $(\mathrm{LiO})^{-}$system, thus accounting for the

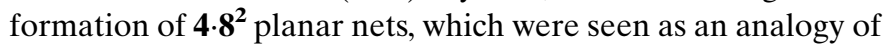
the $\mathbf{6}^{\mathbf{3}}$ graphitic layers; no mention to the ZKC was made though (Sabrowsky et al., 1985).

\section{The phases of $\mathrm{Na}_{2} \mathrm{~S}$ and their related oxides}

Now we shall show that the generalized ZKC can be used to re-interpret and rationalize on a common basis the structures of ordinary compounds, like $\mathrm{Na}_{2} \mathrm{~S}$ and its related oxide $\mathrm{Na}_{2} \mathrm{SO}_{4}$. As is well known, $\mathrm{Na}_{2} \mathrm{~S}$ crystallizes in the antifluorite structure at ambient conditions and undergoes two highpressure phase transitions to cotunnite- and $\mathrm{Ni}_{2} \mathrm{In}$-type structures. It has been suggested that the transition sequence fluorite $\rightarrow$ cotunnite $\rightarrow \mathrm{Ni}_{2}$ In might lead, at higher pressures, to the $\mathrm{MgCu}_{2}$ structure of the cubic Laves phases (Vegas et al., 2001; Vegas \& Jansen, 2002). Although such a transition has never been observed in a binary alloy, it has been observed in related oxides: the well known

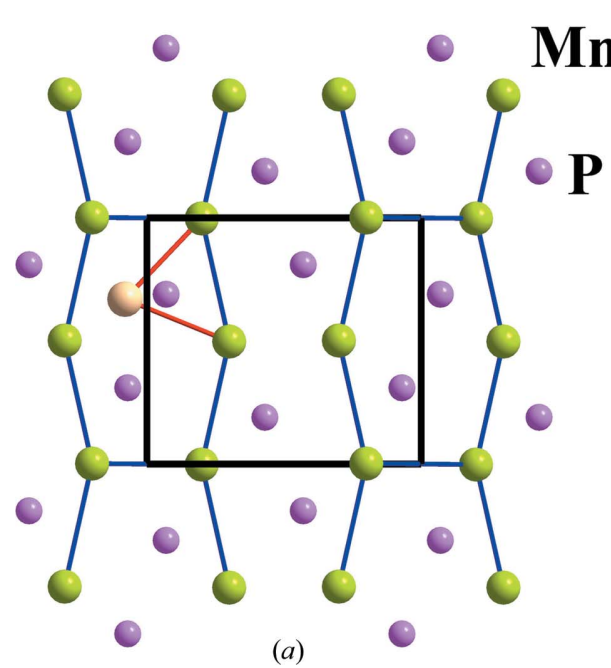

(a)

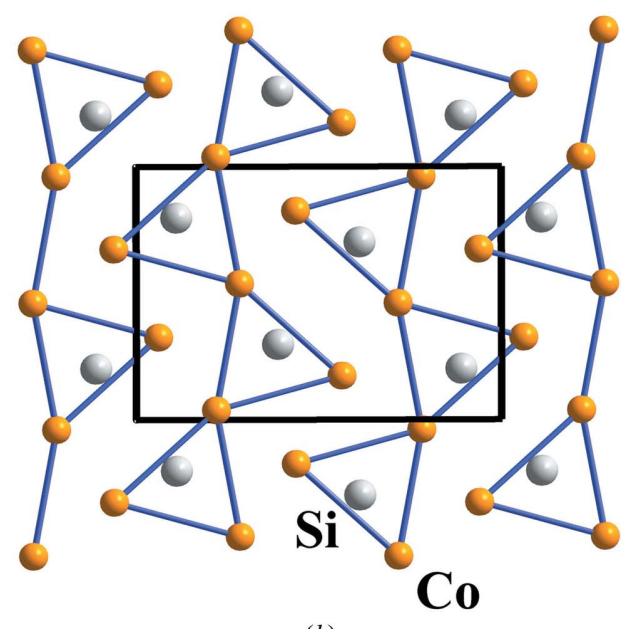

(b)
Figure 3

(a) The $\mathrm{MnP}$ structure projected on the $a b$ plane. The $\mathrm{Mn}$ atoms are green spheres and violet spheres represent the $\mathrm{P}$ atoms. By inserting an additional atom (golden sphere) this structure converts into the $\mathrm{Co}_{2} \mathrm{Si}$-type structure projected in $(b)$. Co atoms form the trigonal prisms and are represented as orange spheres and the $\mathrm{Si}$ atoms as grey spheres. Note that the $\mathrm{P}$ atoms in $(a)$ are equivalent to the $\mathrm{Si}$ atoms in (b). olivine $\left(\mathrm{Ni}_{2} \mathrm{In}\right) \rightarrow$ spinel $\left(\mathrm{MgCu}_{2}\right)$ transition. As far as we know, the only theoretical prediction for this transition refers to $\mathrm{Na}_{2} \mathrm{~S}$ (Schön et al., 2004), which could reach the Laves phase structure at a pressure of the order of 1 Mbar. In any case, according to the generalized $\mathrm{ZKC}$, if we assume that one $\mathrm{Na}$ donates one electron, the BS becomes $(\mathrm{NaS})^{-}$with eight valence electrons. Since the polyanion can be considered isostructural to $\mathrm{ZnS}$, $\mathrm{Na}_{2} \mathrm{~S}$ therefore adopts a stuffed blende structure (as is well known a fluorite structure can be seen as a stuffed blende). This arrangement has been found in the BS of the high-temperature phase of $\mathrm{Li}_{2} \mathrm{SO}_{4}$, which essentially can be regarded as an oxygen-stuffed fluorite. Let us 
recall that $\mathrm{Li}_{2} \mathrm{~S}$ is antifluorite at ambient conditions and it undergoes a phase transition to anti-cotunnite at high pressure (Grzechnik et al., 2000).

Probably the most convincing explanation of what occurs in fluorite-type compounds is exemplified in $\mathrm{Ag}_{2} \mathrm{Te}$. Careful structure refinements (Sakuma \& Saitoh, 1985; Schneider \& Schulz, 1993) lead to a disordered structure (anti-fluorite) in which the Te atom is fixed at $(0,0,0)$ and one $\mathrm{Ag}$ at $8(c)\left(\frac{1}{4}, \frac{1}{4}, \frac{1}{4}\right)$ with an occupation factor of 0.647 (almost four $\mathrm{Ag}$ atoms), while the second $\mathrm{Ag}$ atom is disordered at 32(f) $(x=0.422)$ with an occupation factor of 0.0881 . That is, a blende-type structure is formed by the BS $(\mathrm{AgTe})^{-}$, but the second $\mathrm{Ag}$ atom seems to be rattling around in the structure searching for available electronic charge to stabilize. Similar features are observed in all copper and silver chalcogenides. In the case of $\mathrm{Ag}_{2} \mathrm{~S}$, Tomaszewski (1992) reports the transitions: $P 2_{1} / n \rightarrow$ $(450 \mathrm{~K}) \operatorname{Im} 3 m \rightarrow(860 \mathrm{~K}) \mathrm{Fm} \overline{3} m$. We have not found any structure determination of the $\operatorname{Im} 3 m$ phase. The roomtemperature phase is cotunnite, which exhibits a phase transition to anti-fluorite at $860 \mathrm{~K}$, so the fourfold connectivity is maintained across the phase transitions. The important issue here is that the generalized ZKC explains the BS of these compounds, including that of the cotunnite structure.

Let us emphasize the fact that the cotunnite structure has never been interpreted as a (IV)-(IV)-like structure, but it should within the generalized ZKC by assuming a charge transfer occurs between a pair of atoms of the same species to stabilize octets, leading to the formation of (IV)-(IV) skeletons. As noted earlier in this paper, this view might be questioned at first sight, because it indirectly implies the possible existence/formation of negatively charged cations (cations $e x$ officio). However, several experiments exist that indicate the existence of such entities (e.g. potassides) even in the solid state (Tinkham \& Dye, 1985; Nakayama et al., 1994; Terskikh et al., 2001), so it is conceivable that these ions might coexist in other compounds, regardless of the fact that such entities could be (or not) identified in conventional diffraction experiments.

The previous discussion is relevant because $\mathrm{Na}_{2} \mathrm{~S}$ transforms to cotunnite at high pressures (Vegas et al., 2001). This transition, which takes place in several binary compounds, also occurs in oxides. An interesting example is provided by $\mathrm{Cs}[\mathrm{LiCr}] \mathrm{O}_{4}$, a cotunnite-like compound at room temperature, which undergoes two phase transitions at high temperatures: one to olivine-like (Pnma) at $427 \mathrm{~K}$, and a subsequent transition to anti-fluorite $(F 4 \overline{3} \mathrm{~m})$ at $573 \mathrm{~K}$ (Tomaszewski, 1992).

The most accepted description of cotunnite depicts this structure as a set of trigonal prisms (formed by either $\mathrm{Na}$ atoms in $\mathrm{Na}_{2} \mathrm{~S}$ or LiCs entities in $\mathrm{CsLiCrO}_{4}$ ) that are occupied by the nominal anion. The prisms form zigzag chains, as in $\mathrm{Co}_{2} \mathrm{Si}$ (directly related to those found in the $\mathrm{Ni}_{2} \mathrm{In}$ structure), in which the cation array can be regarded as a stuffed MnPtype structure (Fig. 4). In our example, the $\mathrm{MnP}$ array is formed by the $\mathrm{Cs}(\mathrm{Mn})$ and $\mathrm{Cr}(\mathrm{P})$ atoms. Thus, if $\mathrm{Li}$ is inserted into the structure, the trigonal prisms are formed. Here, the similarity with $\mathrm{Na}_{2} \mathrm{~S}$ is explained by assuming that Cs donates its valence electron, so a $\mathrm{BS}$ of the type ( $\Psi-\mathrm{BeCr})$ is formed, in which $\mathrm{Cr}$ is structurally equivalent to $\mathrm{S}$, as is well known. In the case of $\mathrm{Na}_{2} \mathrm{~S}$, to show a similar behaviour, one must admit some sort of disproportion between the two Na atoms, so that one $\mathrm{Na}$ atom donates its electron to the $\mathrm{BS}(\mathrm{NaS})^{-}$forming a $(\Psi-\mathrm{MgS})$ array.

In any case, the important comparison is that in both $\mathrm{Na}_{2} \mathrm{~S}$ and $\mathrm{CsLiCrO}_{4}$, the arrays correspond to (II)-(VI) compounds, which lead to a (IV)-(IV)-type array typical of the binary alloys. Thus, if we connect the pairs $(\mathrm{Na}-\mathrm{S})$ and $(\mathrm{Li}-\mathrm{Cr})$,

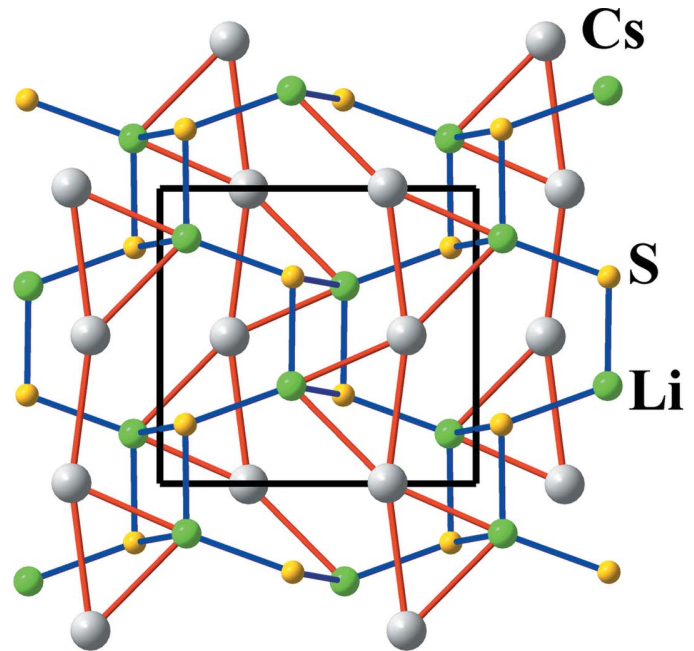

(a)

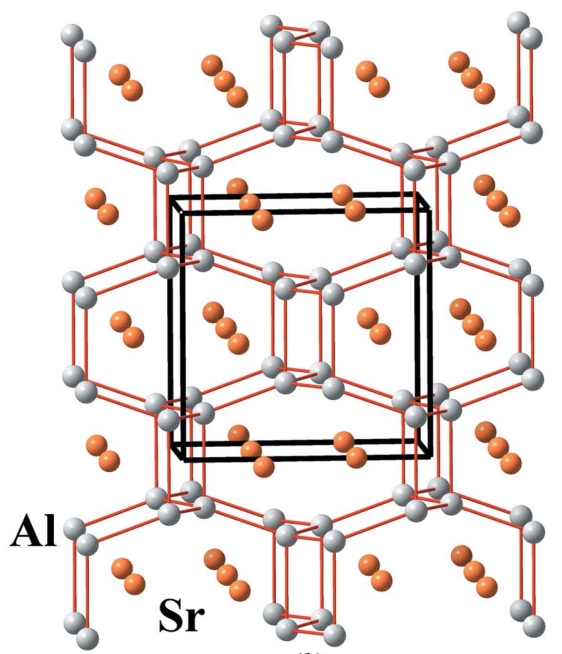

(b)

Figure 4

(a) The $\mathrm{CsLiSO}_{4}$ structure projected on the $a b$ plane. The cation array: Cs (grey), Li (green) and S (orange) form a cotunnite-like structure, in which $\mathrm{Cs}$ and $\mathrm{Li}$ form the trigonal prisms centred by $\mathrm{S}$. The cotunnite structure is outlined by red contacts, to be compared with that shown in Fig. $3(b)$. We have drawn in blue the Li-S contacts to show that they form a four-connected net similar to that of the $\mathrm{SrAl}_{2}$ structure represented in $(b)$. Red contacts connect the $\mathrm{Al}$ atoms (grey spheres). Orange spheres represent $\mathrm{Sr}$ atoms. respectively, a three-dimensional four-connected net is formed. This net corresponds to the Zintl polyanion $\left(\mathrm{Al}_{2}\right)^{2-}(\Psi-\mathrm{Si})$ in the Zintl phase $\mathrm{SrAl}_{2}$ (Fig. 4). Thus, skeletons of either elements or pseudoelements of Group 14 are always obtained. An interesting observation is that these examples represent new cases of how cations undergo their own phase transitions, in spite of the presence of $\mathrm{O}$ atoms (Vegas \& Jansen, 2002).

At still higher pressures, the cotunnite-like $\mathrm{Na}_{2} \mathrm{~S}$ transforms into a hexagonal phase of the $\mathrm{Ni}_{2}$ Intype. To understand this transition we must recall that cotunnite is a filled MnP-type structure, whereas $\mathrm{Ni}_{2}$ In is a filled NiAs-type structure. However, the important issue here is that the $\mathrm{MnP} \rightarrow \mathrm{NiAs}$ transition has been never experimentally observed in $\mathrm{CrS}$, so it is concluded 
that the cotunnite $\rightarrow \mathrm{Ni}_{2}$ In transition is a consequence of the former transition. This necessarily implies that the $\mathrm{MnP} \rightarrow$ $\mathrm{NiAs}$ transition takes place in the BS even in the presence of additional atoms in the structure. In fact, the $\mathrm{MnP}-\mathrm{NiAs}$ phase transition is implicit in the $\mathrm{K}_{2} \mathrm{SO}_{4}$-type $\rightarrow$ olivine-type transition, which takes place, for example, in $\mathrm{K}_{2} \mathrm{SO}_{4}$ itself. Thus, the structures of binary alloys seem to be preserved and the BSs undergo their own phase transitions even in the presence of additional cations or $\mathrm{O}$ atoms. In any case, the interesting fact is that both structures are intimately related and that they are found in many compounds $\left(\mathrm{Na}_{2} \mathrm{~S}, \mathrm{Ni}_{2} \mathrm{Si}, \mathrm{Co}_{2} \mathrm{Si}, \mathrm{Ca}_{2} \mathrm{Si}\right.$, $\mathrm{Ca}_{2} \mathrm{SiO}_{4}, \mathrm{~K}_{2} \mathrm{SO}_{4}, \mathrm{Na}_{2} \mathrm{SO}_{4}$ etc. $)$.

An interesting observation is that the $\mathrm{Ni}_{2} \mathrm{In}$ type is sometimes stable under high-pressure conditions, while in other instances, the $\mathrm{Ni}_{2} \mathrm{In}$ structure is obtained at high temperature, always starting from a cotunnite-type phase. In some cases, like in $\mathrm{Ca}_{2} \mathrm{SiO}_{4}$, the cotunnite-like structure seems to be metastable and, when heated, it converts irreversibly into the $P 6_{3} / m m c \mathrm{Ni}_{2}$ In-type phase. In other cases, as in $\mathrm{K}_{2} \mathrm{SO}_{4}$, the cotunnite-like structure is the stable phase at room temperature; the $\mathrm{Ni}_{2}$ In phase being obtained at high temperature.

An interesting exception is $\mathrm{Na}_{2} \mathrm{SO}_{4}$, for which the cotunnite-like phase is unknown; the room-temperature phase being the mineral thenardite $(F d d d)$. Considering the idea that oxidation can be equivalent to the application of pressure (Vegas \& Jansen, 2002), it should be expected that $\mathrm{Na}_{2} \mathrm{SO}_{4}$ could have stabilized the $\mathrm{MgCu}_{2}$ structure, by adopting the spinel structure (it should be noted that the arrangement of atoms in spinel $\mathrm{MgCu}_{2}$ is also adopted by $\mathrm{Si}$ in the highpressure spinel $\mathrm{Si}_{3} \mathrm{~N}_{4}$ ). However, the sulfate presents the thenardite structure, where the $\mathrm{BS}\left(\mathrm{Na}_{2} \mathrm{~S}\right)$ is of the $\mathrm{TiSi}_{2}$-type. Although this structure has no directed bonding, it presents some remarkable features which are worth discussing. The first striking feature is that cations adopt the structure of a compound where atoms of the Group 14 are involved. The second feature is that the $\mathrm{S}$ atoms (equivalent to $\mathrm{Ti}$ in $\mathrm{TiSi}_{2}$ ) form a diamond-like array similar to that which is found in spinels. The third characteristic is that the $\mathrm{Si}$ ( $\mathrm{Na}$ atoms in $\mathrm{Na}_{2} \mathrm{SO}_{4}$ ) atoms form fragments of the Al-subarray in the $\mathrm{MgAl}_{2} \mathrm{O}_{4}$ spinel or $\mathrm{Si}$ in $\mathrm{Si}_{3} \mathrm{~N}_{4}$. All these features clearly indicate that the structure of thenardite is halfway between the olivine and the spinel structures. So it could be inferred that the $\mathrm{TiSi}_{2}$ structure is an intermediate in the $\mathrm{Ni}_{2} \mathrm{In} \rightarrow$ $\mathrm{MgCu}_{2}$ transition. Consequently, thenardite might well be an intermediate structure in the olivine $\rightarrow$ spinel transition which takes place in the earth mantle. Although one may think that this reasoning would be somewhat controversial as the complete transition olivine $\rightarrow$ thenardite $\rightarrow$ spinel has never been observed, it must be pointed out that the complete transition path can be reconstructed from the observed transitions in two closely related compounds. Thus, let us consider the transition thenardite $\rightarrow$ olivine which takes place at high temperature in $\mathrm{Na}_{2} \mathrm{SO}_{4}$. Subsequently, this path is completed with the transition spinel $\rightarrow$ thenardite which takes place at high temperature in $\mathrm{Na}_{2} \mathrm{MoO}_{4}$. Thus, the following general scheme is proposed

$$
\text { spinel } \underset{\mathrm{Na}_{2} \mathrm{MoO}_{4}}{\longrightarrow} \text { thenardite } \underset{\mathrm{Na}_{2} \mathrm{SO}_{4}}{\longrightarrow} \text { olivine }
$$

At this point, the thermal behaviour of thenardite merits a special comment, because when it is heated three phases with a cation array of the $\mathrm{Ni}_{2}$ In-type appear, namely $\mathrm{Cmcm}$ (filled $\mathrm{CuSO}_{4}$-type), Pnma (olivine) and the $P 6_{3} / m m c$ phase. The third one is, in fact, an oxygen-stuffed $\mathrm{Ni}_{2} \mathrm{In}$ alloy, the other two phases being orthorhombic distortions of the former. To the best of our knowledge, $\mathrm{Na}_{2} \mathrm{SO}_{4}$ is the only compound that presents these three phases. Thus, when thenardite is heated the following sequence is found: Fddd $\rightarrow \mathrm{Cmcm} \rightarrow$ Pnma $\rightarrow$ $P 6_{3} / m m c$. In the case of $\mathrm{Ag}_{2} \mathrm{SO}_{4}$, only one transition has been reported: $\mathrm{Fddd} \rightarrow \mathrm{Pb}_{3} / \mathrm{mmc}$. In the three high-temperature phases the structural features are quite similar: their cation arrays are of the $\mathrm{Ni}_{2} \mathrm{In}$-type. Moreover, the hexagonal phase is just that of the high-pressure phase of $\mathrm{Na}_{2} \mathrm{~S}$ (also $\mathrm{Pb}_{3} / \mathrm{mmc}$ ). It should be noted that whereas the graphite-like layers are irregular in the orthorhombic phases, they are completely regular in the hexagonal variant. Such regularity is also true of the same rotational disorder observed in the oxygen-stuffed anti-fluorites. Thus, the $\mathrm{XO}_{4}^{2-}$ anions $\left(\mathrm{SO}_{4}^{2-}, \mathrm{SiO}_{4}^{2-}\right)$ are completely disordered in the $\mathrm{Pb}_{3} / \mathrm{mmc}$ phases. The reason for this could be that in the orthorhombic phases the layers $(\mathrm{NaS}$, $\mathrm{CaSi}, \mathrm{AgS}$ ) show $\mathrm{Na}-\mathrm{S}$ contacts of different lengths, which would correspond to hypothetical single/double bonds. However, in $P 6_{3} / m m c$, all the distances are equal, mimicking an aromatic ring configuration. In these conditions each $\mathrm{Na}-\mathrm{S}$ contact (with 1.5 electrons on average) does not fulfill the stability conditions found in the other phases, as oxygen tends to share a pair of electrons. The fact that disorder is not only a thermal effect is exemplified by $\mathrm{ZnSO}_{4}$. At room temperature this compound is $\mathrm{VCrO}_{4}$-type, but transforms into a highcristobalite structure at $973 \mathrm{~K}$ (Spiess \& Gruehn, 1979), in which the $\mathrm{ZnS}$ subarray recovers the blende structure and where the $\mathrm{O}$ atoms are not disordered but fixed near the midpoint of the $\mathrm{Zn}-\mathrm{S}$ bonds. Let us recall that the $P 6_{3} / m m c$ structure of $\mathrm{Na}_{2} \mathrm{SO}_{4}$ is stable (with disorder at $520 \mathrm{~K}$ ) at lower temperatures ( $450 \mathrm{~K}$ below) than $\mathrm{ZnSO}_{4}$. In the case of the related compound $\mathrm{GaPO}_{4}$ (Haines et al., 2006) thermal disorder is induced at $1303 \mathrm{~K}$ with the $\mathrm{O}$-atom density forming a continuous ring around the vector joining neighbouring $\mathrm{Ga}$ and $\mathrm{P}$ atoms. This type of disorder, however, is closer to the image of fixed $\mathrm{O}$ atoms near the bond pairs than the rotational disorder observed in both high-temperature phases of $\mathrm{Na}_{2} \mathrm{SO}_{4}$ and $\mathrm{Li}_{2} \mathrm{SO}_{4}$.

\section{Concluding remarks}

The above examples demonstrate that the generalization of the ZKC allows for a universal definition of a crystal structure in terms of the general formula $D_{n}[\mathbf{B S}] X_{m}$ involving pseudoatoms. The universal principle we introduce here just needs information about the constituent atoms and on the known phases of the $p$-block elements, which can exist at ambient conditions, or under high-pressure/temperature conditions. We believe this is a novel, powerful tool to understand crystal 
structures. In addition, the principle can be applied to predict the structure of complex compounds from simpler ones, e.g. a given $A B$ skeleton is the building block for $A_{2} B, A B_{2}, A B X_{m}$, ... compounds of a very different kind. The low-temperature phase $\alpha-\mathrm{NaCoPO}_{4}$ provides a good example of this. In this structure, the CoP subarray is of the NiAs type, because the $\mathrm{Na}$ atom provides one electron to the $\mathrm{P}$ atom converting $\mathrm{CoP}$ into a $\Psi$-(CoS) skeleton (also NiAs-type). The striking feature is that this array is maintained in $\mathrm{NaCoPO}_{4}$. Thus, the $\mathrm{CoS}$ structure remains when more ( $\mathrm{Na}$ atoms) and more (O atoms) atoms are added into the structure. Interestingly, when this compound is heated, the high-temperature phase $\beta-\mathrm{NaCoPO}_{4}$ adopts a structure that has been related to a stuffed tridymite structure (Hammond \& Barbier, 1996), in which the CoP subarray forms a four-connected net characteristic of (IV)(IV) compounds. Again, such types of transformations, as discussed in the article, are precisely those often observed in binary compounds.

We believe that the principles discussed here serve to rationalize the observed structures and to put them on a general basis. Surely, they will also help to make reliable predictions of the structural features of new crystalline materials. Our interest now is to join the efforts of scientists of different fields to solve the challenges following from these principles, perhaps the high-pressure community and, of course, theorists developing first-principles calculations. We are aware that our contribution is very likely only the first step in future developments, so now it works only at a qualitative level, so new ideas are welcome to quantify the unexpected regularities uncovered here. The ideas introduced throughout this work demonstrate, for the first time, that there exists a univocal link between chemical composition (in terms of pseudoatoms) and structure: the generalized Zintl-Klemm concept.

We acknowledge financial support from DGI (MEC) under project MAT2004-05867-C03-02 and from Comunidad Autónoma de Madrid under project CM-UCM-910481.

\section{References}

Borisov, S. V. (2000). Crystallogr. Rep. 45, 709-713.

Fischer, C. C., Tibbetts, K. J., Morgan, D. \& Ceder, G. (2006). Nature Mater. 5, 641-646.

Grzechnik, A., Vegas, A., Syassen, K., Loa, I., Hanfland, M. \& Jansen, M. (2000). J. Solid State Chem. 154, 603-611.
Haines, J., Cambon, O., Prudhomme, N., Fraysse, G., Keen, D. A., Chapon, L. C. \& Tucker, M. G. (2006). Phys. Rev. B, 73, 014103.

Hammond, R. \& Barbier, J. (1996). Acta Cryst B52, 440449.

Hart, G. L. W., Blum, V., Walorski, M. J. \& Zunger, A. (2005). Nature Mater. 4, 391-394.

Jansen, M. (2002). Angew. Chem. Int. Ed. Engl. 41, 37473766.

Karpov, A., Nuss, J., Wedig, U. \& Jansen, M. (2004). J. Am. Chem. Soc. 126, 14123-14128.

Kauzlarich, S. M. (1996). Editor. Chemistry, Structure, and Bonding of Zintl Phases and Ions. New York: VCH.

Marqués, M., Flórez, M., Recio, J. M., Santamaría, D., Vegas, A. \& Baonza, V. G. (2006). J. Phys. Chem. B, 110, 18609-18618.

Nakayama, H., Klug, D. D., Ratcliffe, C. I. \& Ripmeester, J. A. (1994). J. Am. Chem. Soc. 116, 9777-9778.

Oganov, A. R. \& Glass, C. W. (2006). J. Chem. Phys. 124, 244704.

O'Keeffe, M. \& Hyde, B. G. (1985). Structure and Bonding, Vol. 61, pp. 77-144. Berlin: Springer Verlag.

Parthé, E. \& Chabot, B. (1990). Acta Cryst. B46, 7-23.

Parthé, E. \& Engel, N. (1986). Acta Cryst. B42, 538-544.

Sabrowsky, H., Mertens, P. \& Thimm, A. (1985). Z. Naturforsch. B, 40, 122-123.

Sakuma, T. \& Saitoh, S. (1985). J. Phys. Soc. Jpn, 54, 36473648.

Santamaría-Pérez, D. \& Vegas, A. (2003). Acta Cryst. B59, 305323.

Santamaría-Pérez, D., Vegas, A. \& Liebau, F. (2005). Structure and Bonding, Vol. 118, pp. 121-177, Berlin: Springer Verlag.

Schneider, J. \& Schulz, H. (1993). Z. Kristallogr. 203, 1-15.

Schön, J. C., Cancarevic, Z. \& Jansen, M. (2004). J. Chem. Phys. 121, 2289-2304.

Schön, J. C. \& Jansen, M. (1996). Angew. Chem. Int. Ed. Engl. 35, 1287-1304.

Seifert-Lorenz, K. \& Hafner, J. (2002). Phys. Rev. B, 66, 094105.

Simak, S. I., Häußermann, U., Abrikosov, I. A., Eriksson, O., Wills, J. M., Lidin, S. \& Johansson, B. (1997). Phys. Rev. Lett. 79, 13331336.

Spiess, M. \& Gruehn, R. (1979). Z. Anorg. Allg. Chem. 456, 222240.

Terskikh, V. V., Moudrakovski, I. L., Ratcliffe, C. I. \& Ripmeester, J. A. (2001). J. Am. Chem. Soc. 123, 2891-2892.

Tinkham, M. L. \& Dye, J. L. (1985). J. Am. Chem. Soc. 107, 61296130.

Tomaszewski, P. E. (1992). Phase Transitions, 38, 127-220.

Vegas, A. (2000). Crystallogr. Rev. 7, 189-286.

Vegas, A., Grzechnik, A., Syassen, K., Loa, I., Hanfland, M. \& Jansen, M. (2001). Acta Cryst. B57, 151-156.

Vegas, A. \& Jansen, M. (2002). Acta Cryst. B58, 38-51.

Vegas, A., Santamaría-Pérez, D., Marqués, M., Flórez, M., Baonza, V. G. \& Recio, J. M. (2006). Acta Cryst. B62, 220-227.

Zheng, C. \& Hoffmann, R. (1989). Inorg. Chem. 28, 1074-1080.

Zintl, E. \& Dullenkopf, W. (1932). Z. Phys. Chem. Abt. B, 16, 195205. 